Available online on 15.11.2020 at http://ujpr.org
Universal Journal of Pharmaceutical Research
An International Peer Reviewed Journal
Open access to Pharmaceutical research is an open access article distributed under the terms of the Creative Commons Attribution-Non
Commercial Share Alike 4.0 License which permits unrestricted non commercial use,
provided the original work is properly cited
Volume 5, Issue 5, 2020

\title{
THE PRACTICE AND PERCEPTION OF HOSPITAL PHARMACISTS TOWARDS ERRORS IN DISPENSING MEDICINES AND THEIR POSSIBLE CAUSES IN OMDURMAN MILITARY HOSPITAL, SUDAN

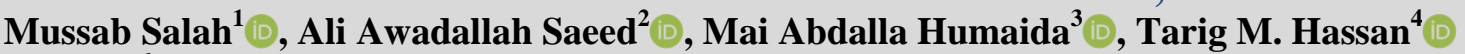 \\ ${ }^{I}$ Hospital pharmacy, Faculty of clinical and industrial pharmacy, National University, Sudan. \\ ${ }^{2}$ Department of pharmacology and therapeutics, Pharmacy program, Napata college, Sudan. ${ }^{3}$ Pharmacy practice department, Faculty of clinical and industrial pharmacy, National University, Sudan. ${ }^{4}$ Clinical pharmacy department, Faculty of clinical and industrial pharmacy, National University, Sudan.
}

\section{ABSTRACT}

Introduction: Hospital pharmacies dispensing errors are common and investigating them for identifying factors involved in it and developing strategies to minimize their occurrence. Errors can arise at any stage during the dispensing process. Dispensing errors were identified by checking the prescribed drug against the dispensed medication.

Materials and Methods: A cross sectional study involving 100 pharmacists who were administered a survey research designed to assess pharmacists' attitudes, factors associated with DEs and involvement in DE, conducted between $1^{\text {st }}$ January 2019 and $1^{\text {st }}$ February 2019 at Omdurman Military Hospital (OMH) Pharmacies. A data analyzed by Statistical Package for Social Sciences software version 21 .

Results: $55 \%$ from the pharmacists in the study have poor attitude toward dispensing errors. The most common factors influencing dispensing errors as stated by participants were lack of therapeutic training (stated by $81 \%$ ), $62 \%$ from the participants stated that workload and time pressure are causes of dispensing errors in area of factors associated with the work environment. $48 \%$ from the pharmacists in the study committing dispensing errors, $41.7 \%$ from them committed dispensing errors once while $23 \%$ committed fourth or more.

Conclusion: With the multiplicity of risk factors in our environment, there is urgent need to reinforce the training of pharmacists and the provision of resource materials and enabling work environment aimed at minimizing medication errors.

Keywords: Cross sectional study, dispensing errors, Omdurman Military Hospital, pharmacist.

Article Info: Received 8 September 2020; Revised 7 October; Accepted 3 November, Available online 15 November 2020

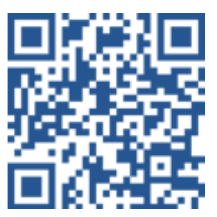

\section{Cite this article-}

Mussab Salah M, Saeed AA, Humaida MA, Hassan TM. The practice and perception of hospital pharmacists towards errors in dispensing medicinesand their possible causes in Omdurman Military Hospital, Sudan. Universal Journal of Pharmaceutical Research 2020; 5(5):12-16.

DOI: https://doi.org/10.22270/ujpr.v5i5.480

Address for Correspondence

Ali Awadallah Saeed, Department of pharmacology and therapeutics, Pharmacy program, Napata college, Sudan, Tel: +249 0912320825, E-mail: alimhsd@gmail.com

\section{INTRODUCTION}

Hospital pharmacies dispensing errors are common and investigating them for identifying factors involved in it and developing strategies to minimize their occurrence ${ }^{1}$. The core function of pharmaceutical care is dispensing medication and approximately 900 million medicines are dispensed each year by hospital and community pharmacies ${ }^{7}$. Errors can occur at any stage during the dispensing process. 1Before medication is supplied to patients; pharmacists detect $85 \%$ of errors ${ }^{1}$. Undetected errors may occur and may cause serious patient harm and may develop to death $^{9,12}$. To safeguard the quality and safety of patient care by reviewing data on dispensing errors by pharmacists so that risk-reduction strategies are developed $^{1}$. Depending on the dispensing system, research method, and classification of dispensing error types dispensing error rates can be varied between countries. Dispensing the wrong medicine, dispensing the wrong drug strength, and dispensing the wrong dosage form were the most frequent dispensing errors reported. High workload, low staffing, mix-up of lookalike/sound-alike drugs, lack of knowledge/experience, distractions/interruptions, and communication problems within the dispensary team were the most common factors associated with dispensing errors ${ }^{2}$. Checking the prescribed drug against the dispensed 
medication can identify the dispensing errors ${ }^{14}$, this parallel to study conducted by Alaa and et al which found that $97.4 \%$ from pharmacists checked drug name in study conducted in Khartoum ${ }^{17}$. According to Beso et al14dispensing errors were classified into types and subtypes, with adaptations for the medication use $^{3}$. Mainstay of health care is the medication and the risks of drug therapy and prevalence of adverse effects have increased due to an increased number of medication errors $^{1,3}$. The high risk group is the pediatric population and the number of potential adverse drug events generally is higher in inpatients pediatric than inpatients adult population. $4-6{ }^{4}$. $13.2 \%$ prescribing and $19.1 \%$ administration error rate found in study to investigate incidence and type of prescribing and administration errors in pediatric patients in five United Kingdom hospitals. 6Possibility of errors in children compared to adult patients increased due to need to calculate doses according to the age, weight, and body surface area of children, ${ }^{8,9}$.

Ambiguous, incomplete, or confusing prescriptions may result in incorrectly understanding drug prescriptions which lead dispensing and administration problems of drugs ${ }^{10,13}$. Action plans and mechanisms proposed by researchers, health care professionals, and institutions to decrease medication errors and increase patient safety ${ }^{4}$. The source of medication errors and potential adverse drug events (ADEs) is the hospital pharmacy's medication dispensing process ${ }^{2,3}$. Only intercept $33 \%$ of serious medication dispensing errors can be intercepted by nurses before medication administration, so many of these errors could reach patients. Hospitalized patients are more susceptible to harm from dispensing errors because they are critically ill. Many previous studies of dispensing error rates reflect pharmacy dispensing patterns before the widespread use of automated dispensing cabinets ${ }^{5,9}$, which are now commonly deployed by central hospital pharmacies to better control the distribution of frequently used medications on patient care units ${ }^{5}$. Pharmaceutical care main function is the dispensing and approximately 900 million medicines are dispensed each year by pharmacies either community or hospital across England and Wales.1Dispensing is a complex process under pharmacist supervision of the pharmacist $1^{1,2}$. Pharmacy staff manually selecting medication from shelves, transferring the correct number of medication dose units to a container and/or labeling the assembled product represent the dispensing $^{3}$ but automated dispensing systems has been widely advocated to improve efficiency, maximize storage capacity and minimize dispensing errors ${ }^{4,5}$. Consequently, automated dispensing systems are becoming increasingly commonplace in hospital and community Pharmacies across the world ${ }^{4,8,6}$. This study aimed to analyze the frequency and potential causes of dispensing errors in the hospital pharmacy of Omdurman Military Hospital, Khartoum State, 2019

\section{METHODS}

A cross sectional study involving 100 pharmacists in $\mathrm{OMH}$ pharmacies selected by simple random sampling technique using a data collection form which designed to assess pharmacists' attitudes, factors associated with DEs and their involvement in DE.

\section{Study area}

The study conducted between 1st January 2019 and 1st February 2019 at Omdurman Military Hospital (OMH) Pharmacies located in Omdurman.

Data analysis:

Data analyzed using Statistical Package for Social Sciences software, version 21.0 (IBM SPSS Inc., Chicago, IL).

Table 1: Perception toward key factors that influence dispensing errors.

\begin{tabular}{lc}
\hline Factors influence dispensing error & $\begin{array}{c}\text { Percent of } \\
\text { participants }\end{array}$ \\
\hline Lack of therapeutics training & 81 \\
Inadequate drug knowledge and experience & 77 \\
Inadequate knowledge of patient & 49 \\
Inadequate perception of risk & 51 \\
Overworked or fatigued health care professionals & 65 \\
Physical and emotional health issues & 37 \\
Poor communication between health care professional & \\
and with patient & 85 \\
Patient characteristics & 58 \\
Complexity of clinical case including multiple health & \\
conditions Poly pharmacy and high risk & 63 \\
Workload and time pressures & 62 \\
Distraction and interruptions & 53 \\
Lack of standardized protocols and procedures & 76 \\
Insufficient resources & 56 \\
Issues with the physical work environment & 25 \\
Naming of medicines & 54 \\
Labeling and packaging & 37 \\
Repetitive systems for ordering processing and & 34 \\
authorization & \\
Patient monitoring & 48 \\
\hline
\end{tabular}




\section{RESULTS}

In demographic data of participants:57\% aged less than 25 years old while others aged 25 years and more, $80 \%$ were female while $20 \%$ were male, $40 \%$ experienced less than 2 years while the others more than 2 years.

In area of attitude variables, high errors ratings were observed in dimensions of: committing dispensing errors (43\% sometimes), reporting dispensing errors (37\% rarely), supervising interns/ technician on dispensing errors (44\% sometimes while $21 \%$ rarely), higher work pressure increase chance to DE (53\% from pharmacists stated that usually it increase it), inter professional relations (31\% from pharmacists stated that it was rarely), availability of information, structural and environmental effects. Clarification of difficult prescriptions with colleagues, use of technologies to reduce DE and inadequacy of staff for $\mathrm{t}$ existing workload were rated low, all of which have implications for aggravating prescribing errors. Total $45 \%$ from pharmacist in the study had good attitude while $55 \%$ had poor attitude (Figure 1).

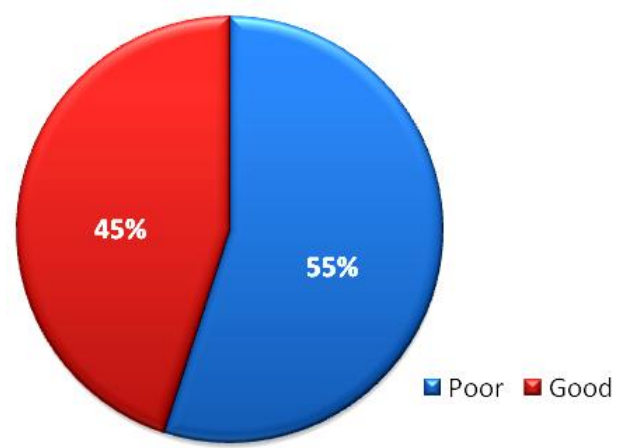

Figure 1: Attitude score of the pharmacists toward dispensing errors.

Health care professional's factors that may influence medication errors more than $50 \%$ of the pharmacist in the study stated that lack of therapeutic training $(81 \%)$, inadequate drug knowledge $(77 \%)$, inadequate perception of risk $(51 \%)$, overworked or fatigued healthcare professionals $(65 \%)$ and poor communications between healthcare professionals and with the patients $(85 \%)$ were the most important factors in this area. In the area of factors associated with patients that may influence medication errors more than $58 \%$ from pharmacists in the study stated that patient's characteristics $(58 \%)$ and complexity of clinical case $(63 \%)$ were the factors in this area. In the area of factors associated with the work environment more than $53 \%$ from the pharmacists in the study stated that workload and time pressure (62\%), distractions and interruptions by staff and patients (53\%), lack of standardized protocols and procedures (76\%) and insufficient resources $(56 \%)$ were the most important factors in this area. In the area of factors associated with medicines $54 \%$ from the pharmacists in the study stated that naming of medicines was the most important factor while $37 \%$ stated that labelling and packaging of medicines was the other factor. On the other hand, in factors associated with tasks less than
$50 \%$ from the pharmacists in the study stated that repetitive systems for ordering, processing and authorization (34\%) and patients monitoring (48\%) were the factors in this area can lead to dispensing errors (Table 1).

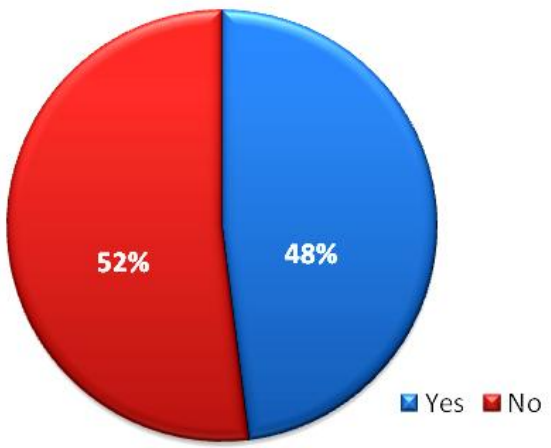

Figure 2: The rate of committing dispensing errors among pharmacist participants.

$48 \%$ from pharmacist participants stated that they get prescription with dispensing errors while other (52\%) didn't get them (Figure 2). Total $41.7 \%$ from participants stated that number of dispensing errors per prescription was one, $23 \%$ stated that was four or more, $18.8 \%$ stated that was three and $16.7 \%$ stated that the number of dispensing errors was two (Figure 3 ).

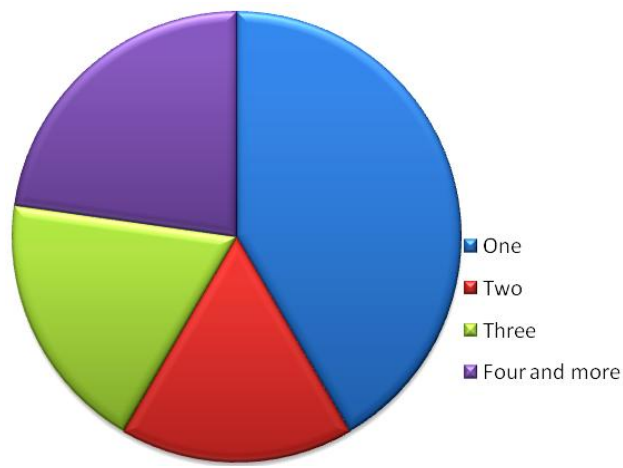

Figure 3: Rate of dispensing errors among pharmacist participants.

In area of type of dispensing errors $60 \%$ of pharmacist stated that it was content type, $30 \%$ stated that labeling dispensing errors and $10 \%$ from pharmacists stated that documentation errors (Figure 4).

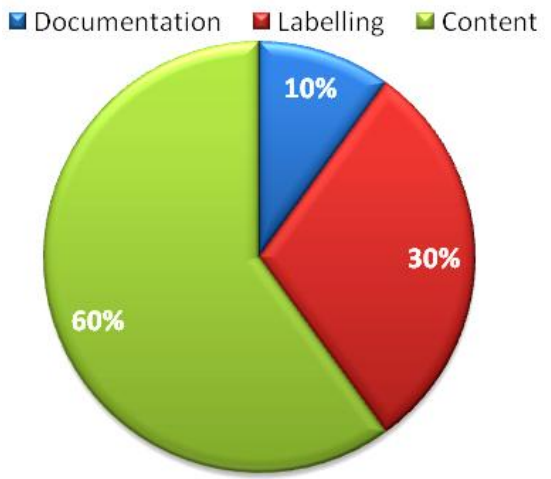

Figure 4: Types of dispensing errors among pharmacist participants. 


\section{DISCUSSION}

In area of attitude variables, high errors ratings were observed in dimensions of: committing dispensing errors (43\% sometimes), reporting dispensing errors (37\% rarely), supervising interns/technician on dispensing errors (44\% sometimes while $21 \%$ rarely), higher work pressure increase chance to DE (53\% from pharmacists stated that usually it increase it), inter professional relations ( $31 \%$ from pharmacists stated that it was rarely), availability of information, structural and environmental effects. Clarification of difficult prescriptions with colleagues, use of technologies to reduce DE and staff inadequacy for the existing workload were rated low, all of which have implications for aggravating prescribing errors. The attitudes of the pharmacists in the study concluded in that $55 \%$ from the pharmacists in the study have poor attitude toward dispensing errors, in a similar survey in Tasmania most of the pharmacists believed that the risk of dispensing errors is increasing and they suggested interventions for improvement like having a mechanism for dispensing procedures checking, checking the original prescription when dispensing repeats, keeping pharmacist knowledge up to date and counseling patient at the time of supply ${ }^{18}$.

In the area of Health care professional's factors that may influence dispensing errors, $81 \%$ of the pharmacist in the study stated that lack of therapeutic training influence in dispensing errors, this may indicate that workplace training during undergraduate, and internship program may play a role to overcome this factor. $77 \%$ of participants stated that inadequate drug knowledge may cause dispensing errors, which can be solved by encouraging pharmacists to enroll in continuing professional educations during practice. $51 \%$ of participants stated that inadequate perception of risk may be a cause of dispensing errors, and this may be due to the lack of knowledge about wrong medicines hazardous effects to patients. $65 \%$ of participants stated that overload of work cause stress and fatigue to health care professionals leading to prescribing and dispensing errors, which need more attention from the director of the health facility to take care about the work schedule. $85 \%$ of participants indicated that poor communications between healthcare professionals and with the patients lead to error in prescribing and dispensing medicines, so the availability of counseling area in the pharmacy is very important for the pharmacist -patient communication to overcome any mistake, on the other hand communication with health care professionals is essential in providing efficient pharmaceutical care this could be through regular meetings and discussion with health professionals in the health facility.

In the area of factors associated with the work environment more than $62 \%$ from the participants in the study stated that workload and time pressure are causes of dispensing errors, $53 \%$ of them stated that distractions and interruptions by staff and patients may lead to dispensing errors, while $76 \%$ of the pharmacists indicated that lack of standardized protocols and procedures cause dispensing errors and $56 \%$ of them mentioned the insufficient resources are reasons for dispensing errors, this parallel with Avery A et al14 and Slight SP et al., ${ }^{15}$ studies except in the area of physical work environment e.g. lighting, only $25 \%$ from pharmacists in our study stated that physical work environment was a factor involved in medication errors. Generally organizing the work place and providing suitable environment in the hospital pharmacy have a great effect in reducing dispensing errors, especially proper lightening, temperature, and adequate counter spaces that facilitate the workflow. Dispensing medication to patients through a window by the pharmacy technician will help in minimizing distraction in the hospital pharmacy while the pharmacist provides patient consultation in the consultation area ${ }^{17}$. Also the availability of standard operating protocols and procedures will improve the quality of the pharmaceutical service, and maintain proper guidance for junior pharmacists to follow.

In area of factors associated with patients in dispensing errors, $58 \%$ of participants in the study stated that the patient's characteristic's influence the occurrence of dispensing errors, that angry and nervous patients those came to the pharmacy made distraction and interrupted the pharmacist work. Also the complexity of clinical case (63\% of participants) in which the prescription might contain more than 3 medicines, lead to pharmacist confusion. In the area of factors associated with medicines $54 \%$ from the pharmacists in the study stated that naming of medicines was the most important factor while $37 \%$ stated that labeling and packaging of medicines was the other factor, this parallel to the work done by Avery A et al., ${ }^{14}$ and Slight SP et al., ${ }^{15}$ studies except in the factor of labeling and packaging of medicines which is important. On the other hand, in factors related to assignments less than $50 \%$ of the pharmacists in the study reported that repeated ordering, processing and authorization regimes (34\%) and patient monitoring $(48 \%)$ were factors in this area that could lead to dispensing errors. These errors parallel the study by Lynette Jamesa et al., ${ }^{2}$ in their sixty investigation papers that identified factors of dispensing errors. $48 \%$ from the pharmacists in the study committing dispensing errors, $41.7 \%$ from them committed dispensing errors once while $23 \%$ committed fourth or more. $60.4 \%$ from pharmacists committed dispensing errors stated that, the dispensing errors in the content while $43.8 \%$ committed dispensing errors in labeling.

\section{CONCLUSION}

With the multiplicity of risk factors in our environment, there is urgent need to reinforce the training of pharmacists and the provision of resource materials and enabling work environment aimed at minimizing medication errors.

\section{RECOMMENDATIONS}

Pharmacists should play a more dynamic role in minimizing dispensing errors through higher commitment and alertness, self - updating and 
organizing training sessions, for the therapeutic benefit to the patients. Inter-professional team work should be encouraged to minimize errors.

\section{ACKNOWLEDGEMENTS}

Authors acknowledge the pharmacists participated in the study from Omdurman military hospital.

\section{AUTHOR'S CONTRIBUTION}

Mussab Salah and Ali Awadallah Saeed: made the hypothesis and participate in manuscript writing Mai Abdalla Humaida and Tarig M. Hassan: Participate in manuscript writing.

\section{CONFLICT OF INTEREST}

No conflict of interest associated with this work

\section{REFERENCES}

1. Aldhwaihi K, Schifano F, Pezzolesi C, Umaru N. A systematic review of the nature of dispensing errors in hospital pharmacies. Integ Pharm Res Pract 2016; 5:1-10. http://dx.doi.org/10.2147/IPRP.S95733

2. James KL, Barlow D, McArtney R, Hiomc S, Roberts D, Whittlesea C. Incidence, type and causes of dispensing errors: a review of the literature. Int J Pharm Pract 2009, 17:9-30. PMID: 20218026

3. Silva MDG, Rosa MB, Franklin BD, et al. Concomitant prescribing and dispensing errors at a Brazilian hospital: a descriptive study. Clinics (Sao Paulo) 2011; 66(10):16911697. http://dx.doi.org/10.1590/S1807-59322011001000005

4. Mohammad Ali Cheragi, Human Manoocheri, et al. Types and causes of medication errors from nurse's viewpoint. Iranian J Nurs Midwif Res 2013; 18(3):228-231. PMID: 23983760

5. Cina JL, Gandhi TK, Churchill W, et al. How many hospital pharmacy medication dispensing errors go undetected? J Qual Patient Saf 2006; 32(2): 73-80. http://dx.doi.org/10.1016/s1553-7250(06)32010-7

6. Jean Spinks, Jackson J, Kirkpatrick CM, et al, Disruptive innovation in community pharmacy-Impact of automation on the pharmacist workforce. Res Social Adm Pharm 2016; 13(2): 1-6.http://dx.doi.org/10.1016/j.sapharm.2016.04.009
7. RAbduelkarem A. Extending the role of pharmacists in patient care: are pharmacists in developing nations ready to change. Pharmacol Pharm 2014;5(09):986-875. http://dx.doi.org/10.4236/pp.2014.59097

8. Cheung KC, Bemt PMLA, Bouvy ML, et al. Medication incidents related to automated dose dispensing in community pharmacies and hospitals - a reporting system study. PLOS ONE 2014; 9 (7):e101686. http://dx.doi.org/10.1371/journal.pone.0101686

9. Shawahna R, Abbas A, Ghanem A. Medication transcription errors in hospitalizedpatients settings: a consensual study in Palestinian nursing practice. BMC Health Serv Res 2019; 19, 644. https://doi.org/10.1186/s12913-019-4485-3

10. Velo GP, Minuz P. Medication errors: prescribing faults and prescription errors. British J Clin Pharmacol 2009; 67(6):624-628 https://doi.org/10.1111/j.1365-2125.2009.03425.x

11. Knudsen P, Herborg H, Mortensen AR, Knudsen M, Hellebek A. Preventing medication errors in community pharmacy: root-cause analysis of transcription errors BMJ Qual Saf 2007; 16(4): 285-290. https://doi.org/10.1136/qshc.2006.022053

12. Alan F Merry. How does the law recognize and deal with medical errors. J Royal Soc Med 2009; 102(7):265-271. https://doi.org/10.1258/jrsm.2009.09k029

13. Gelayee DA, Mekonnen GB. Perception of community pharmacists towards dispensing errors in community pharmacy setting in gondar town, Northwest Ethiopia, Bio Med Res Int 2017; Article ID 2137981, 9pages. https://doi.org/10.1155/2017/2137981

14. Anum Saqib, Atif M, Ikram R, et al, Factors affecting patients' knowledge about dispensed medicines: A Qualitative study of healthcare professionals and patients in Pakistan, PLoS One 2018; 13(6):e0197482. https://doi.org/10.1371/journal.pone.0197482

15. Saqib A, Atif M, Ikram R, et al. Factors effective on medication errors: A nursing view. J Res Pharm Pract 2013; 2(1):18-23.https://doi.org/10.4103/2279-042X.114084

16. Abdelaziz A, Awad MM. Assessment of pharmacist dispensing practices and their educational role toward patients on oral cytotoxic drugs regimen in RICK. Sudan, J Pharm Pract Comm Med 2019; 5(4):63-68. https://doi.org/10.5530/jppcm.2019.4.17

17. Nair RP, Kappil D, Tonja M. 10 strategies for minimizing dispensing errors, Pharmacy Tmes. 2010-01-2003:13:11

18. Peterson GM, Bergin WJK. Pharmacist's attitudes towards dispensing errors: their causes and prevention. J Clin Pharm Therap 2006; 24:1.

https://doi.org/10.1046/j.1365-2710.1999.00199.x 